\title{
METHODS
}

\section{Comparison of PCR-Based Mutation Detection Methods and Application for Identification of Mouse Sult1a1 Mutant Embryonic Stem Cell Clones Using Pooled Templates}

\author{
Boris Greber, Helena Tandara, Hans Lehrach, and Heinz Himmelbauer* \\ Max-Planck-Institute of Molecular Genetics, Berlin, Germany \\ Communicated by Richard G.H. Cotton
}

Reverse genetic approaches to generate mutants of model species are useful tools to assess functions of unknown genes. Recent work has demonstrated the feasibility of such strategies in several organisms, exploiting the power of chemical mutagenesis to disrupt genes randomly throughout the genome. To increase the throughput of gene-driven mutant identification, efficient mutation screening protocols are needed. Given the availability of sequence information for large numbers of unknown genes in many species, mutation detection protocols are preferably based on PCR. Using a set of defined mutations in the Hprt 1 gene of mouse embryonic stem (ES) cells, we have systematically compared several PCR-based point mutation and deletion detection methods available for their ability to identify lesions in pooled samples, which is a major criterion for an efficient large-scale mutation screening assay. Results indicate that point mutations are most effectively identified by heteroduplex cleavage using CEL I endonuclease. Small deletions can most effectively be detected employing the recently described "poison" primer PCR technique. Further, we employed the CEL I assay followed by conventional agarose gel electrophoresis analysis for screening a library of chemically mutagenized ES cell clones. This resulted in the isolation of several clones harboring mutations in the mouse Sult1a1 locus, demonstrating the high-throughput compatibility of this approach using simple and inexpensive laboratory equipment. Hum Mutat 25:483-490, 2005. (c) 2005 Wiley-Liss, Inc.

KEY WORDS: mouse models; mutagenesis; mutation detection; poolability; Sult1a1; Hprt1

DATABASES:

Hprt1 - GenBank: NM013556.1

Sult1a1 - GenBank: L02331.1

www.genome.ucsc.edu/cgi-bin/hgBlat (Mouse Blat Search)

\section{INTRODUCTION}

Chemical mutagenesis is a powerful means of disrupting gene functions. Facing a large number of genes with unknown functions in model organisms such as the mouse, large-scale $N$-ethyl-Nnitrosourea (ENU) mutagenesis programs have gained recent interest [Brown and Balling, 2001; Nadeau et al., 2001]. In species other than the mouse, chemical mutagenesis may be the only feasible way to knock out genes, due to the lack of alternative methodology to manipulate the germline. Classical forward genetic screens, however, have the general disadvantage that the site of a given lesion causing a phenotype needs to be mapped in a tedious process of Mendelian crossing. So-called gene-driven approaches are based on the specific screening of archives of chemically mutagenized samples (gDNA or cDNA) in genes of interest. Upon identification of a mutation, the respective signal can be traced back to the corresponding individual and a living mutant is selectively generated, with the underlying mutation in the particular gene known. Several approaches that fall into this category have been published for a range of organisms. These include Arabidopsis [McCallum et al., 2000], C. elegans [Jansen

(C)2005 WILEY-LISS, INC et al., 1997], Drosophila [Bentley et al., 2000], zebrafish [Wienholds et al., 2003], rat [Smits et al., 2004; Zan et al., 2003], and mouse [Coghill et al., 2002; Vivian et al., 2002]. As in the case of Drosophila, zebrafish, and rat, the library of chemically mutagenized animals can be kept as living stocks, taking samples for mutational screenings, but if possible the individuals may be preserved in the form of seeds (Arabidopsis), frozen animals (C. elegans), sperm (mouse), or embryonic stem (ES) cells (mouse).

Genomic DNA as the primary template for mutation screening offers the advantage of dealing with relatively robust material

Received 10 September 2004; accepted revised manuscript 9 December 2004

*Correspondence to: Dr. Heinz Himmelbauer, Max-Planck-Institute of Molecular Genetics, Ihnestr. 73, D-14195 Berlin, Germany.

E-mail: himmelbauer@molgen.mpg.de

Grant sponsor: German Genome Research Network (NGFN); Grant sponsor: Federal Ministry of Education and Research (BMBF) BioProfile Potsdam/Berlin; Grant number: 0313028B.

DOI: $10.1002 /$ humu.20168

Published online in Wiley InterScience (www.interscience.wiley.com). 
without being dependent on expression of the gene of interest. cDNA on the other hand offers the possibility to cover the complete coding sequence of a gene in a single PCR amplicon. Generally, a mutation detection procedure suitable for screening a library of mutated PCR samples must be capable of identifying the lesions at least in a two-fold dilution. This is because amplicons rather than plasmid clones are to be screened and autosomal mutations are typically heterozygous. Time and expense can be saved if the sensitivity of the screening method allows the pooling of several samples, ideally even before amplifying the gene sequences. Moreover, poolability of samples prior to PCR aids to also save template material, which is an important aspect when dealing with cDNA samples based on RNA isolation in a multiwell format.

A popular method for point mutation detection in PCR amplicons is denaturing HPLC (dHPLC) [O'Donovan et al., 1998]. Prior to sample loading, PCR fragments are heat-denatured and reannealed to form hetero- and homoduplexes in case of a heterozygous sequence change. Under partially denaturing conditions, elution times of the heteroduplexes differ from the homoduplex ones, resulting in an extra peak of ultraviolet (UV) absorbance. The same principle of mutation visualization is exploited by temperature gradient capillary electrophoresis [Li et al., 2002], allowing for the paralleled analysis of samples. An alternative approach to detect mutations in heteroduplex fragments is based on mismatch cleavage, which can either be performed chemically [Cecchi et al., 1997] or enzymatically [Del Tito et al., 1998]. In this context, heteroduplex cleavage via CEL I endonuclease from celery presents a technically undemanding and highly specific mutation detection assay. The enzyme identifies all types of single basepair changes and small deletions, resulting in two digestion products that add up in size to the original heteroduplex fragment [Oleykowski et al., 1998]. A third approach for detecting sequence alterations in PCR products is matrix assisted laser desorption/ionization (MALDI) resequencing [Stanssens et al., 2004]. Briefly, forward and reverse strands of PCR amplicons are selectively transcribed in vitro, enabled by the incorporation of T7 and SP6 promoter sequences into the $5^{\prime}$ ends of the primers. In separate reactions, both transcripts are digested in a controlled way with RNAses, e.g., specifically cutting after C and $U$ residues, respectively. The fragmentation products are then analyzed by MALDI-time of flight (TOF) mass spectrometry. Mutations become apparent as specific peaks in the spectra in comparison with the wild type.

Deletions present another important type of mutations. They may occur at the genomic as well as at the transcriptional level. For PCR-mediated mutation detection, deletions must be in the PCR-compatible range. This requirement is particularly fulfilled for deletions at the transcript level, which most commonly arise from genomic point mutations in the canonical splice sites and lead to the skipping of single exons during mRNA splicing. Deletion mutations of this kind represent a significant portion of ENU-induced mutations in the mouse [Chen et al., 2000; Justice et al., 1999], but are also estimated to account for about $15 \%$ of all disease-causing mutations in humans [Krawczak et al., 1992]. Focusing on the detection of splice mutations at the cDNA level, the simplest mutation detection procedure would be to separate the mutant RT-PCR products from the wild-type ones according to their differences in size. This could be achieved by conventional gel electrophoresis or, in an automated way, by fragment analysis on a capillary electrophoresis system using fluorescently-labeled primers for amplification. It can be envisaged that the sensitivity of fragment analysis would be limited by the amount of PCR product that can be loaded onto the system. To overcome this obstacle, the wild-type signal can be suppressed in the course of a modified nested PCR using primers that span the predicted site of the deletion. The suppression of the wild-type signal is realized by adding to the first amplification reaction a third primer, binding to the wild-type template but not to the mutant within the deleted region. For the wild-type, a short fragment is then preferentially formed by this so-called "poison" primer and the corresponding external oligonucleotide, preventing the formation of wild-type product in the subsequent nested PCR reaction. In other words, the deleted product can be formed without any competing effects by wild-type product generation. This principle is applicable to the RT-PCR-based detection of splice mutations as well as to small genomic deletions, e.g., induced by trimethylpsoralen (TMP) together with low-energy UV light in C. elegans [Edgley et al., 2002]. In mammals, however, small genomic deletions appear to account only for a minor portion in the TMP/UV mutational spectrum [Greber et al., 2003].

\section{MATERIALS AND METHODS Isolation and Characterization of Hprt1-Deficient ES Cell Clones}

Male mouse ES cells were grown according to standard procedures [Hogan et al., 1994]. In order to induce genomic mutations, ES cells were chemically mutagenized by treatment with $1.5 \mathrm{mM} \mathrm{N}$-ethyl-N-nitrosourea for $90 \mathrm{~min}$ at $37^{\circ} \mathrm{C}$ in suspension. Clones deficient in activity of the X-linked Hprt 1 gene (HPRT1; MIM\# 308000) were isolated as previously described [Greber et al., 2003]. Briefly, cells surviving the exposure to mutagen were cultivated for 10 days to allow for HPRT1 protein depletion in clones harboring functional mutations in the corresponding gene. Then, a subset of the growing cells was replated onto gelatin-coated dishes to be subjected to negative selection with 6 -thioguanine $(10 \mu \mathrm{M})$. After a further 10 days of selection, colonies were picked and expanded. RNA was isolated using the Trizol reagent (Invitrogen; www.invitrogen.com) following the manufacturer's instructions. Reverse transcription was performed using reverse transcriptase from Moloney murine leukemia virus (Amersham; www.amersham.com) and oligo-dT priming, following the company's protocol. Using cDNA as template and primers shown in Table 1, Hprt1 PCR reactions were carried out essentially as previously described [Greber et al., 2003], except that a touchdown protocol and $5 \mathrm{U}$ of a 1:10 mixture of homemade Pfu and Taq DNA polymerases was employed for amplification. Mutations were identified by sequencing of purified Hprt1 RT-PCR fragments using standard dyeterminator technology on ABI Prism 3100 instrumentation (Applied Biosystems; www.appliedbiosystems.com).

\section{Mutation Detection With Model ES Cell Mutant Clones}

Dilution series of Hprt1 mutant clones against the wild-type were typically prepared at the cDNA level. As required only for dHPLC and CEL I analysis, PCR products were denatured at $95^{\circ} \mathrm{C}$ for $5 \mathrm{~min}$ and cooled down to room temperature in a thermocycler with a decrease of $0.5^{\circ} \mathrm{C}$ per minute to enable heteroduplex formation. dHPLC was performed with unpurified PCRs on a Transgenomic (www.transgenomic.com) WAVE system. Melting profiles of the fragments analyzed (Table 1) were calculated by means of the manufacturer's software. All samples were run at three different temperatures. Optimal temperatures were $56^{\circ} \mathrm{C}$ for the c.84T $>$ A mutation and $57^{\circ} \mathrm{C}$ for the c.407T $>$ A lesion. The acetonitrile concentration of the elution buffer was increased from 14.5 to $17.5 \%(\mathrm{v} / \mathrm{v})$ and from 14 to $16.5 \%$, respectively, within a time range of $2 \mathrm{~min}$. The c.203T $>\mathrm{G}$ mutation could not be 
TABLE 1. RT-PCR Amplicons Used for Mutation Analysis*

\begin{tabular}{|c|c|c|c|c|c|}
\hline Fragment name & Size [bp] & Primer sequences & Used for & Including mutation & position in PCR fragment \\
\hline Hprt-int & 593 & $\begin{array}{l}\text { fwd: CGTCGTGATTAGCGATGATG } \\
\text { rev: CGTGATTCAAATCCCTGAAGTAC }\end{array}$ & All but dHPLC & $\begin{array}{l}\text { c. } 84 \mathrm{~T}>\mathrm{A}, \\
\text { c. } 203 \mathrm{~T}>\mathrm{G}, \\
\text { c. } 407 \mathrm{~T}>\mathrm{A}, \\
\Delta \operatorname{ex} 3, \Delta \operatorname{ex} 6\end{array}$ & $\begin{array}{c}64,184 \\
387 \\
115-298,383-465\end{array}$ \\
\hline dHPLC-I & 336 & $\begin{array}{l}\text { fwd: CGTCGTGATTAGCGATGATG } \\
\text { rev: CCACCAATAACTTTTATGTCCC }\end{array}$ & dHPLC & $\begin{array}{c}\text { c. } 84 T>A, \\
\text { c. } 203 \mathrm{~T}>\mathrm{G}, \\
\Delta \operatorname{ex} 3\end{array}$ & $\begin{array}{l}64,184 \\
115-298\end{array}$ \\
\hline dHPLC-II & 297 & $\begin{array}{l}\text { fwd: GGGACATAAAAGTTATTGGTGG } \\
\text { rev: CGTGATTCAAATCCCTGAAGTAC }\end{array}$ & dHPLC & $\begin{array}{c}\text { c. } 407 \mathrm{~T}>\mathrm{A} \\
\Delta \operatorname{ex} 6\end{array}$ & $\begin{array}{c}73, \\
69-151\end{array}$ \\
\hline ex3-poison & 797 & $\begin{array}{l}\text { fwd: CTTACCTCACTGCTTTCCGG } \\
\text { rev: CTGGCAACATCAACAGGACTC } \\
\text { psn: CAAGGGGGGCTATAAGTTCTTTG }\end{array}$ & $\begin{array}{l}\text { First "poison" } \\
\text { primer PCR }\end{array}$ & $\Delta e x 3$ & $223-406$ \\
\hline
\end{tabular}

* The Hprt1-int primers define a 593 bp fragment starting at position+21 (referring to the open reading frame) of the Hprt1 RefSeq cDNA sequence (NM_13556.1). This amplicon was split into two parts for dHPLC analysis. The forward and reverse primers for the first "poison" primer PCR lie outside the Hprt1 coding sequence whereas the interfering primer itself (psn) corresponds to a portion of exon 3 . The point mutations indicated in the fifth column refer to the protein coding sequence. The positions of the corresponding lesion sites in the PCR fragments generated are listed in the last column.

TABLE 2. Comparison of PCR-Based Mutation Detection Methods

\begin{tabular}{|c|c|c|c|c|c|}
\hline & $\begin{array}{c}\text { Max. } \\
\text { pooling } \\
\text { ratio mutant } \\
\text { vs. } \mathbf{w t}^{\mathrm{a}}\end{array}$ & $\begin{array}{c}\text { Max. } \\
\text { fragment } \\
\text { length }>\text { or } \\
<500 \text { bp }\end{array}$ & $\begin{array}{c}\text { Signal } \\
\text { information } \\
\text { content }\end{array}$ & $\begin{array}{l}\text { Simplicity } \\
\text { robustness }\end{array}$ & Affordability ${ }^{c}$ \\
\hline dHPLC & $1 / 5$ & $<$ & + & ++ & + \\
\hline $\begin{array}{l}\text { MALDI } \\
\text { re-sequencing }\end{array}$ & $1 / 5$ & $\leq$ & +++ & ++ & + \\
\hline CEL 1 & $1 / 10^{b}$ & $>$ & ++ & +++ & $++^{d}$ \\
\hline $\begin{array}{l}\text { Capillary } \\
\text { electrophoresis }\end{array}$ & $1 / 15$ & & & & \\
\hline \multirow{2}{*}{$\begin{array}{l}\text { "Poison" primer } \\
\text { PCR }\end{array}$} & $<1 / 100$ & $>$ & + & ++ & ++ \\
\hline & & $>$ & ++ & ++ & ++ \\
\hline
\end{tabular}

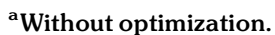

bWith agarose electrophoresis.

${ }^{c}$ Without instrumentation.

${ }^{\mathrm{d}}$ If using home-made enzyme.

The dashed line separates methods for the detection of point mutations (above) and deletions (below)

detected by this method. CEL I reactions were performed at $42^{\circ} \mathrm{C}$ for $20 \mathrm{~min}$ in a final volume of $15 \mu \mathrm{l}$ containing 4-(2. hydroxyethyl)-1-piperazine ethane sulfonic acid) (HEPES) $(\mathrm{pH}$ $7.0 ; 10 \mathrm{mM}), \mathrm{MgSO}_{4}(10 \mathrm{mM}), \mathrm{KCl}(10 \mathrm{mM})$, Triton X-100 $(0.002 \% \mathrm{v} / \mathrm{v})$, bovine serum albumin (BSA) $(0.2 \mu \mathrm{g} / \mathrm{ml}), 200 \mathrm{ng}$ of crude PCR product, and CEL I enzyme isolated from celery, essentially as described elsewhere [Yang et al., 2000]. Reactions were initiated by the addition of DNA template and terminated by adding $5 \mu \mathrm{l}$ of $75 \mathrm{mM}$ EDTA. Samples were immediately analyzed by agarose gel electrophoresis and ethidium bromide staining. To some extent, the optimal amount of enzyme appeared to be dependent on the size of the PCR product digested, in that larger fragments required more enzyme. We therefore titrated the enzyme and considered a given CEL I concentration as optimal if the original amount of DNA template was reduced by about one-third as a result of unspecific cutting events leading to a background smear on the $2 \%(\mathrm{w} / \mathrm{v})$ agarose gels. MALDI resequencing was carried out at Methexis Genomics NV (Zwijnaarde, Belgium), as described elsewhere [Stanssens et al., 2004]. Different from the other approaches, samples were pooled only after reamplification, which was performed to incorporate T7 and SP6 promoter sequences at the $5^{\prime}$ ends of the forward and reverse strands, respectively. In vitro transcribed RNA from the forward and reverse strands was cleaved after $U$ and $C$ residues in four separate reactions prior to MALDI-TOF analysis. Fragment analysis by capillary electrophoresis was carried out on MegaBACE instrumentation (Amersham Biosciences) using fluorescein isothiocyanate (FITC)-labeled primers for amplification and ethanol precipitation for purification of the Hprt1 fragments. Poison primer PCRs to detect the $\Delta$ ex 3 deletion were performed employing the ex3-poison oligonucleotides shown in Table 1 in a first reaction. Nested PCRs employing the Hprt1-int primers were done on a 1:1 $\times 10^{4}$ dilution of the first reaction.

\section{Mutant ES Cell Archive Generation and Sult1a1 Pilot Screen}

ENU mutation frequencies were based on 6-thioguaninemediated negative selection as described above and were defined as the number of 6-TG-resistant colonies divided by the total number of colonies under selection. Spontaneous mutation events were negligible in the ENU concentration range investigated. A library of mutant clones was established from ES cells mutagenized at a frequency of approximately 1 in 2,500 (1.5 mM ENU for 90 

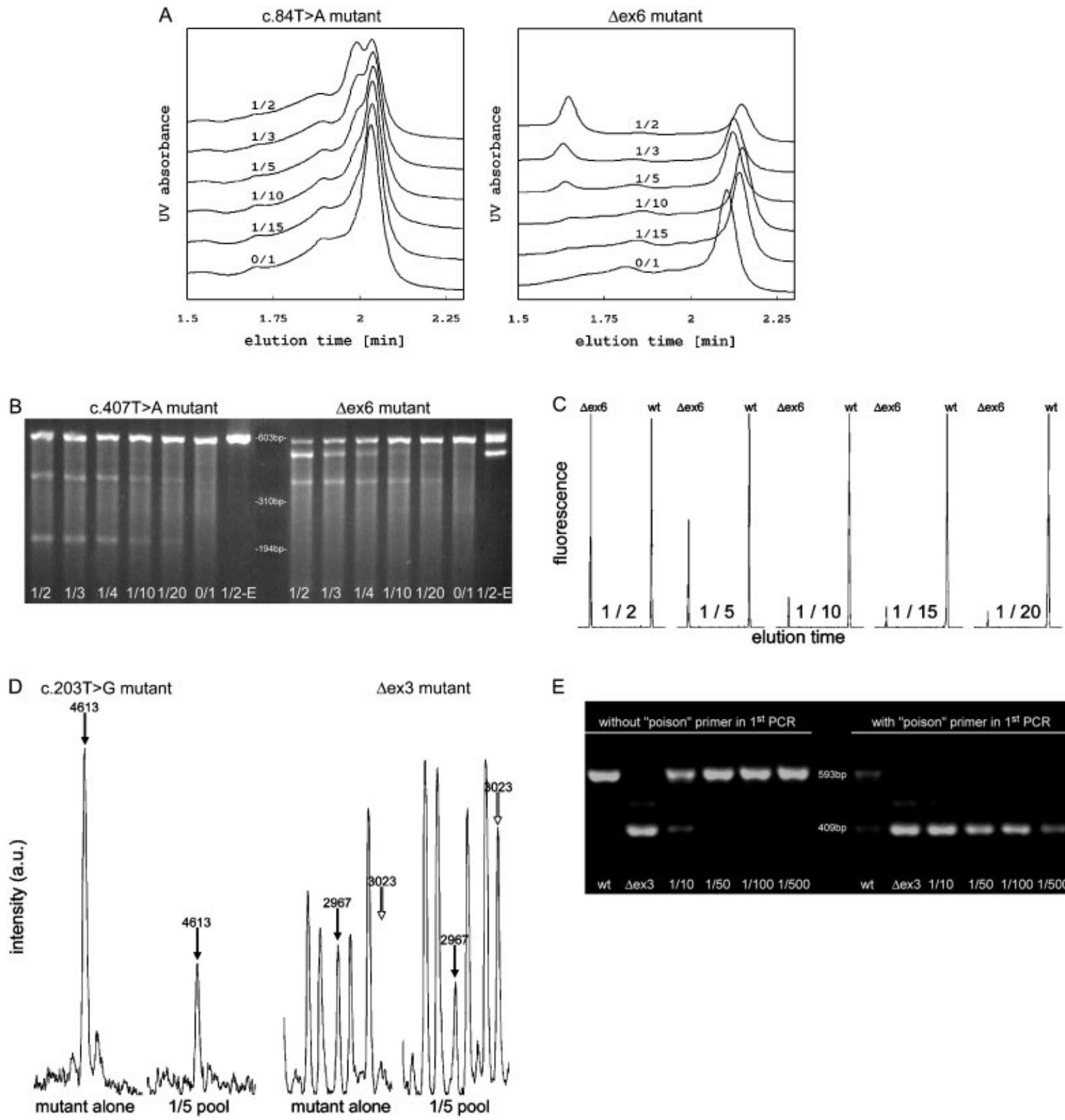

E

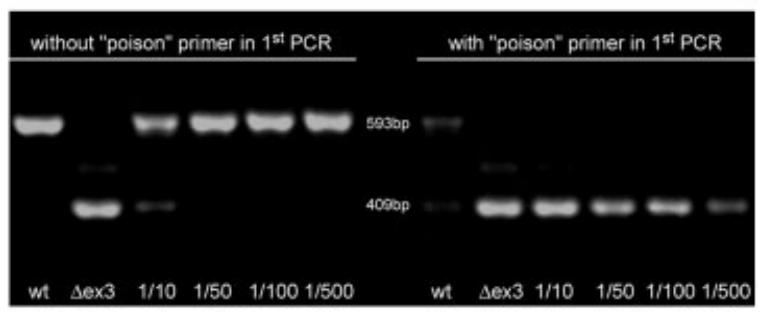

FIGURE 1. Detection of mouse Hprt1 mutations using different PCR-based screening approaches. Dilution series were prepared of mutant against wild-type cDNA to evaluate the sensitivity of each method. The different graphs show representative results for individual mutations from the set of clones investigated, i.e., results were similar within each group (point mutations or exon deletion clones). A: Examples of point mutation (left chart) and deletion detection (right) by dHPLC. The heteroduplex peak for the c.84T $>$ A point mutation quickly disappears with decreasing mutant content in the PCR template mixture. Deletion detection with dHPLC appears to be slightly more sensitive. B: Agarose gel with partial CEL I heteroduplex digests. The c.407T $>$ A mutation is cleaved at position 387 of the 593 bp PCR fragment generating two digestion products (see Table 1). In case of an exon deletion (right panel) there are superimposing effects of heteroduplex cleavage and simple size separation due to the deletion. Pooling ratios are indicated at the bottom; 1/2-E: lack-of-enzyme control. C: Analysis of FITC-labeled Dex6 and wild-type fragments using capillary electrophoresis. Note the excellent signal-to-noise ratio of this method. D: Relevant parts of MALDI-TOF spectra analyzing in vitro transcribed forward-stranded RNA that was fragmented after each U-residue. Spectra are presented for the c.203T $>G$ point mutation (left) and the $\Delta$ ex 3 deletion clone (right), showing signals for the mutant alone and the corresponding 1/5 pool against the wild type, respectively. Analysis is based on diagnostic peaks that appear as a consequence of the mutation. The filled arrows point at such mutant-specific signals, whereas the peak at 3,023 Da (right panel) is unique to the wild type as compared to the $\Delta$ ex 3 spectrum. Numbers denote fragment masses in Daltons. E: Nested PCR with (right panel) and without (left) an exon-3-specific "poison" primer included in the first amplification reaction. Using the additional primer, the wild-type band is almost completely suppressed whereas the mutant band is still present, even at high dilutions. 


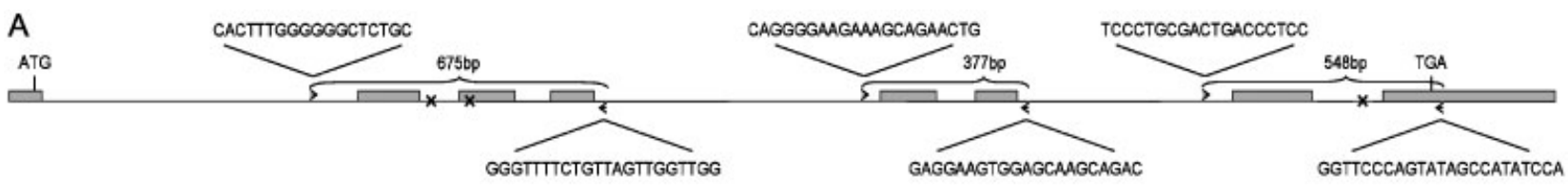

\section{B}

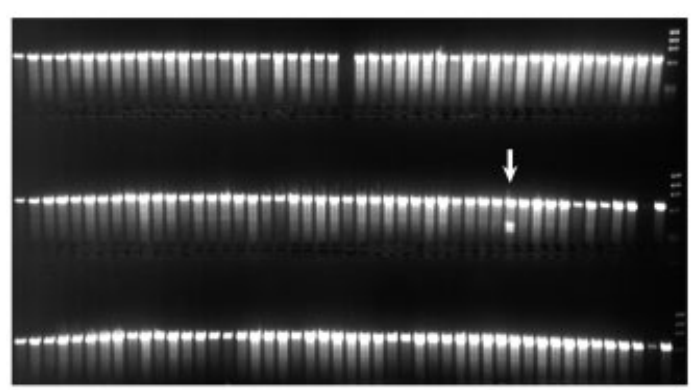

C

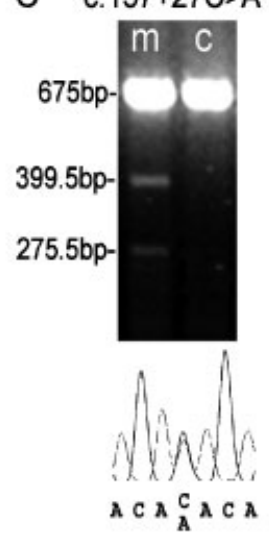

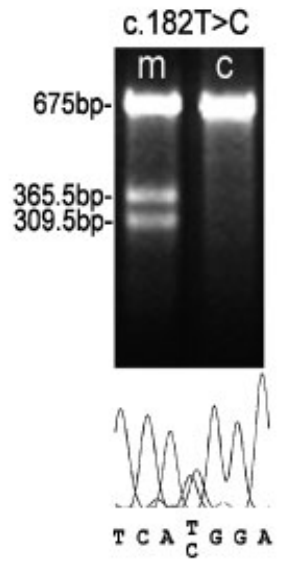

c. $784+116 \mathrm{G}>\mathrm{A}$

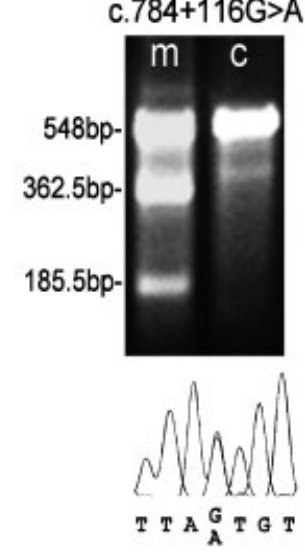

FIGURE 2. CEL I-based pilot screen of an archive of ENU-treated mouse ES cells for mutations in the Sult1a1 locus. A: Target gene structure as derived from a Blat search against the mouse genomic sequence using the Sult1a1 cDNA sequence (L02331.1) as input. Boxes indicate individual exons. The major portion of the coding sequence was amplified from DNA samples of ENU-treated ES cells in three separate PCR reactions employing the primers indicated. To screen for the presence of mutations, products were treated with CEL I endonuclease and analyzed by agarose gel electrophoresis. B: Agarose gel with 144 out of 2,304 samples screened for mutations in the 675-bp amplicon. The arrow points at a positive sample. C: Mutations identified in the Sult1a1 screen. The upper panel shows partial CEL I digests of PCR products amplified from isolated mutant clones $(\mathrm{m})$. Control fragments $(\mathrm{c})$ were not cleaved. The presence of the mutations and their expected positions-according to the CEL I cleavage product pattern-were confirmed by sequencing of the mutant amplicons, respectively (lower panel). The locations of the three mutations identified are also marked ("x") in part $\mathrm{A}$. The mutation nomenclature refers to the $\mathrm{A}$ of the translation initiation codon.

min at $37^{\circ} \mathrm{C}$ ). Treated cells were pipetted into 24 96-well plates giving rise to $4.1 \pm 2.1$ (average \pm standard deviation [SD]) colonies per well. After a growth period of one week, aliquots of the cell samples were replated and the master plates were frozen for single-clone recovery. Upon confluence of the replica plates, genomic DNA was isolated following a crude extraction protocol [Matise et al., 2000]: PBS-washed cells were lysed overnight at $55^{\circ} \mathrm{C}$ in $50 \mu \mathrm{l}$ of $10 \mathrm{mM}$ Tris- $\mathrm{Cl}(\mathrm{pH} 7.5), 10 \mathrm{mM} \mathrm{NaCl}, 10 \mathrm{mM}$ EDTA, $0.5 \%(\mathrm{w} / \mathrm{v}) \mathrm{SDS}$, and $0.1 \%(\mathrm{w} / \mathrm{v})$ proteinase $\mathrm{K}$, in a humidified atmosphere. DNA was then precipitated for $1 \mathrm{hr}$ by adding $100 \mu \mathrm{l}$ of ice-cold $75 \mathrm{mM} \mathrm{NaCl}$ in ethanol. The DNA sticking to the bottom of the wells was washed twice with $70 \%$ $(\mathrm{v} / \mathrm{v})$ ethanol, air-dried, and finally dissolved in $200 \mu \mathrm{l}$ of $1 \times \mathrm{TE}$ buffer. A total of $1 \mu \mathrm{l}$ of each preparation was used as template for subsequent $25 \mu \mathrm{l}-\mathrm{PCR}$ reactions.

Amplifications of parts of the Sult1al locus were performed by nested PCR on a 3,405-bp amplicon generated using primers $5^{\prime}$ CTACACAAAGATCCCTACCACTGAG- ${ }^{\prime}$ (fwd) and $5^{\prime}$ CAGTGTTAGGACTGATGGCTTTC-3' (rev), which match to exons 1 and 8, respectively. Denaturation and reannealing of PCR fragments followed by CEL I treatment was carried out as described above. The digests were analyzed using large homemade agarose gel electrophoresis chambers with a capacity of 384 samples. Fragments were separated across a distance of approximately $6 \mathrm{~cm}$.

\section{RESULTS}

\section{Comparison of PCR-Based Mutation Detection Methods}

In the course of our own efforts to establish a suitable screening protocol for libraries of ENU-treated mouse ES cells, we have systematically evaluated PCR-based mutation detection methods with respect to their high-throughput compatibility, due to a lack of published comparative data. In order to obtain a set of targeted mutations, we subjected mouse ES cells to chemical mutagenesis using the standard mutagen ENU. Mutations targeted to the Xlinked Hprtl gene were isolated via the 6-thioguanine-based negative selection assay [Hooper et al., 1987]. Clones resistant to 6-thioguanine were characterized by sequencing of the Hprt1 cDNA. From a set of 31 nonredundant clones, mutations in the coding sequence were identified in 29 cases. As expected for ENU, since it is a point mutagen, 23 of the 29 lesions were base substitutions, 14 of which were transversions. The remaining six mutations induced were splice mutations, i.e., single-exon deletions at the transcript level, which were most probably caused by point mutations in the genome interfering with pre-mRNA splicing. Therefore, apart from choosing three base substitutions distributed evenly over the Hprt1 coding sequence, we also selected two exon-skipping mutations for the evaluation of different mutation detection methods. Referring to the open reading frame of the Hprt1 RefSeq cDNA sequence (NM_013556.1), these were the substitutions c.84T $>$ A, c.203T $>\mathrm{G}$, and c.407T $>\mathrm{A}$, as well as deletion clones lacking base pairs 135-318 (exon 3) and 403-475 (exon 6). Fragments containing the lesion sites were amplified from these mutant clones as well as from wild-type ES cells using cDNA as template (Table 1). The mutation detection procedures investigated were dHPLC, CEL I heteroduplex cleavage, MALDI resequencing, capillary electrophoresis, and nested PCR using poison primers in the first amplification reaction.

We generated dilution series of PCR templates from the five model ES cell clones to test the sensitivity of each mutation detection procedure. To obtain realistic information on their 
suitability as high-throughput methods for identifying unknown mutations, the assays were performed without any optimization as to the individual sequence changes. For instance, dHPLC analysis was performed choosing parameters that would allow mutation detection ideally over the whole range of the amplicons and using a minimum elution time, as would be the case when processing large numbers of unknown samples. Representative results for the different methods are shown in Figure 1. Denaturing HPLC could detect all the mutations with the exception of the c.203T $>$ G base change. Sensitivity under conditions optimized for speed of analysis was limited to dilution ratios of about $1 / 5$ (one part mutant PCR template, four parts wild-type), which would allow a pooling of two clones (four alleles) in routine operations. Maximum pooling ratios were similar, when point mutations were compared to deletion detection (Fig. 1A), indicating that sensitivity is actually not so much limited by the resolution capacity of this method but rather by the amount of heteroduplex fragment that is loaded onto the column. Using fluorescence rather than UV detection may increase the sensitivity to some extent by enhancing the specificity of fragment monitoring. Very similar results regarding the detection sensitivity were obtained with MALDI resequencing (Fig. 1D). All of the investigated mutations were detected at pooling ratios of up to $1 / 5$ on average. Mutant-specific signals were hard to distinguish from background noise above this degree of dilution. It is worth mentioning, however, that the signal-to-noise ratio with this method is considerably higher than with conventional sequencing, which could in principle be used for mutation detection as well. CEL Imediated cleavage of heteroduplex fragments is capable of detecting both point mutations and deletions. Using the CEL I assay, all the mutations tested were reliably identified, generating two digestion products of the predicted sizes in each case. Largescale application of this enzyme assay has been reported, analyzing the cleavage products by slab gel electrophoresis and employing differentially-labeled primers [Colbert et al., 2001]. We used simple agarose gel electrophoresis for analysis, achieving similar sensitivities of at least $1 / 10$ (Fig. 1B). Significantly, the cleavage fragment signal intensities did not seem to drop linearly with increasing pooling ratios.

Focusing on the detection of small deletions such as single-exon deletions in cDNAs, fragment analysis by capillary electrophoresis would be a sensitive alternative to analysis by gel electrophoresis, allowing pooling ratios of at least $1 / 15$. Remarkably high signal-tonoise ratios were obtained when using fluorescently-labeled primers (Fig. 1C). To prevent the generation of false positive signals, high-fidelity PCR conditions such as the use of a touchdown protocol and a proofreading DNA polymerase had to be applied. As in the case of dHPLC, detection sensitivity was ultimately limited by the sheer amount of mutant product loaded onto the system. For detecting deletion mutations in pools of samples, it would therefore be desirable to suppress the formation of wild-type product already in the PCR. This can be achieved by performing nested PCRs with a poison primer in the first reaction, which allowed the identification of the mutant form in dilutions of $1 / 100$ and beyond (Fig. 1E), presenting an exceptionally high sensitivity of deletion mutation detection.

\section{CEL I-Based Screen for Induced Genomic Mutations in Mouse ES Cells}

Due to the high sensitivity and reliability of the assay we sought to employ CEL I in a large-scale screen for induced point mutations, based on pooled samples, which has not yet been reported as an application to mouse ES cells. To this end, we mutagenized ES cells with a medium dose of ENU, yielding approximately one functional Hprt 1 mutation per 2,500 clones, as determined by negative selection with 6 -thioguanine. We then generated a library of mutagenized ES cell clones by pipetting ENU-treated cells at a defined dilution into 96-well plates for expansion. The clone set comprised 2,304 samples with a statistical density of $4.1 \pm 2.1$ clones per well (average \pm SD), corresponding to pools of eight haploid genomes at the cellular level. DNA was isolated from replica plates to serve as template for genomic PCR reactions amplifying a target gene of interest.

As a proof-of-principle target, we chose the Sultlal gene, a member of the sulfotransferase 1A gene family, which is the sole mouse ortholog to the three members of the human SULT1A gene cluster (SULT1A1; MIM\# 171150) [Glatt, 2002]. Sulfotransferases, which in human are highly expressed in the gastrointestinal mucosa, play a major role in the elimination of xenobiotics but they may also form chemically reactive, i.e., potentially mutagenic and carcinogenic, metabolites from some substrates [Glatt, 2000]. SULT1A genes show common SNPs among the human population, leading to enzyme variants with altered kinetic properties and stability [Engelke et al., 2000].

We amplified a major portion of the mouse Sult 1al gene 897-bp coding sequence by nested PCR from our ES cell clone set in three separate reactions (Fig. 2A). Heteroduplex formation was enabled by slowly cooling down the heat-denatured PCR fragments. Immediately after CEL I treatment, samples were analyzed by agarose gel electrophoresis, which had previously proven to provide sufficient separation capacity (Fig. 1B). Figure 2B shows an example for the amplicon spanning exons 2-4 of Sult1a1; a positive sample that was identified here is marked by an arrow. Together, we confirmed three positive signals from the primary screen. Since almost all samples of the ES cell archive were represented by several different clones, positive subclones had to be isolated by cultivating the cells from positive positions on culture dishes, followed by picking several colonies each, and expanding these lines in a multiwell plate format. Clonal lines could be established for each of the three mutations, as confirmed by CEL I-based rescreening. The respective PCR products were sequenced, leading to the unequivocal identification of the underlying lesions, i.e., the positions of the heterozygous point mutations were in line with the CEL I digestion pattern of the respective PCR fragments (Fig. 2C; upper panel): Two of the mutations were intronic-L02331:c. 157+27C > A (intron 2); c. $784+116 \mathrm{G}>\mathrm{A}$ (intron 7) - and one was targeted to the Sult1a1 coding sequence-c.182T $>\mathrm{C}$ (exon 3 ) -leading to an amino acid change from Met to Thr at position 61 of the encoded protein (Fig. 2A and $\mathrm{C}$; lower panel).

\section{DISCUSSION}

Most published data regarding the sensitivity of mutation detection is focused solely on one particular method. We have systematically compared several PCR-based mutation detection approaches on the basis of a defined set of lesions. The main focus of these tests was on the detection of mutations in pools of samples while performing the individual assays under conditions optimized for speed of analysis, which are major criteria for mutational screens in a high-throughput format. Representative results for individual clones are presented in Figure 1 and the average sensitivities across all mutations investigated are summarized in Table 2 (first column). Point mutation detection was most sensitive with CEL I endonuclease, using simple agarose gel 
electrophoresis for the analysis of enzyme digests. Regarding dHPLC, there are applications that may allow for the optimization of the elution parameters, thereby enhancing sensitivity. For the detection of unknown point mutations, however, this method was inferior to CEL I. Focusing on the detection of small deletions, the highly sensitive poison primer approach would be the method of choice, enabling the identification of splice mutations at the transcript level or, alternatively, the detection of small deletions in the genome, e.g., following mutagenesis with an effective deletion mutagen [Edgley et al., 2002].

Apart from allowing mutant identification in pools of samples, there are further criteria for a good mutation detection method. These would include the possibility to analyze relatively large fragments and a low degree of false-negative and false-positive signals, as well as inherent simplicity of assays and costeffectiveness, to meet high-throughput demands. General characteristics of the mutation detection methods tested are summarized in Table 2. The maximum sizes of the amplicons to be analyzed without risk of false negative results differ between the individual assays. For point mutation detection, CEL I is superior in this regard to dHPLC or MALDI resequencing, allowing the analysis of amplicons exceeding 500 basepairs. This point is particularly relevant for screening approaches at the cDNA level but also for genomic DNA as template when dealing with species having small introns such as C. elegans. With some of the methods, valuable information can be extracted from the mutation signals, as to the site of the lesion within the analyzed fragment. For example, the accurate sizing of the fragmentation products with MALDI resequencing allowed the unambiguous identification of the underlying mutations in almost all cases with basepair precision (Table 2; third column). With CEL I, the fact that generally two digestion products are formed, adding up in size to the original fragment, reduces the chance of obtaining false positive signals. Moreover, mutations in all five ES cell clones investigated were identified by clear signals like the ones shown in Figure 1B, making heteroduplex cleavage by CEL I a particularly robust assay. Finally, suitability of a given mutation detection method for large-scale applications is also dependent on its costeffectiveness. Apart from inherent factors such as poolability of samples, cost and throughput are dependent on the specific instrumentation used and on the degree of multiparalleled analysis. The latter aspect addresses a particular weakness of dHPLC, which can only process one sample at a time. An improvement in this regard is presented by temperature gradient capillary electrophoresis, for which multicapillary systems are available.

Due to its role of being the most important mammalian model organism, programs have been initiated to ultimately generate mouse mutants for every gene in a gene-driven way [Austin et al., 2004; Auwerx et al., 2004]. Reverse genetic screens, e.g., based on ENU-treated mouse ES cells, will play a role in this context given the fact that a range of genes may not be targeted by alternative approaches. For instance, single-exon genes cannot be disrupted by $5^{\prime}$ gene trap vectors, which are dependent on integration into intronic sequences [Stanford et al., 2001]. Vivian et al. [2002] isolated an allelic series for two nonselectable genes from an archive of highly mutagenized ES cell clones using dHPLC as the primary screening procedure. In a pilot screen, we have applied the CEL I assay to pools of mutagenized clones, resulting in the identification of several induced mutations at the Sultlal locus. The diversity of the point mutations identified (Fig. 2C) underscores the potential of CEL I to cleave all kinds of mismatches in heteroduplex fragments [Oleykowski et al., 1998], while the relatively small number of mutant clones recovered reflects the moderate mutation frequency underlying the ES cell clone archive. Generally, it is advisable not to overload the ES cells with induced lesions, since this may complicate mouse mutant production from targeted clones [Munroe et al., 2000; Vivian et al., 2002]. A second reason for the low number of recovered mutations may reside in the architecture of the ES cell archive; due to the statistical distribution of clones across the individual multiwell positions, about $40 \%$ of these were in samples containing six or more clones (12 or more haploid genomes), as determined by counting growing colonies in about 200 samples. However, the CEL I assay itself has proven to be a suitable screening procedure for DNA pools of mutagenized mouse ES cell clones. Sophisticated slab gel electrophoresis systems visualizing digestion products via fluorescence have been employed in CEL I-based high-throughput screens, allowing pooling ratios of about 1 in 16 [Colbert et al., 2001; Wienholds et al., 2003]. We have achieved somewhat lower, but overall similar, maximum dilution ratios of approximately 1 in 10 using large-scale agarose electrophoresis for analysis. Hence, we think that combining the CEL I assay with such relatively simple instrumentation would generally present a cost-effective alternative that is easy to implement in any laboratory, still providing high performance in many (in particular medium-scale) applications. These would not only include gene-driven screens for induced mutations in model organisms, but could also be extended to the medical field, e.g., in detecting mutations in candidate genes of human disease patients.

\section{ACKNOWLEDGMENTS}

We thank Drs. Patrick Stanssens and Marc Zabeau (Methexis Genomics NV, Zwijnaarde, Belgium) for the MALDI resequencing analysis, Drs. Hans Christian Hennies and Peter Nürnberg (MaxDelbrück-Center for Molecular Medicine, Berlin-Buch, Germany) for running the capillary electrophoresis samples, and Bettina Moser and Dr. Richard Reinhardt (Service Group at MPI for Molecular Genetics, Berlin, Germany) for the dHPLC analysis. We thank Drs. Hans-Rudolf Glatt and Walter Meinl for valuable discussions on the biology of mouse sulfotransferase genes.

\section{REFERENCES}

Austin CP, Battey JF, Bradley A, Bucan M, Capecchi M, Collins FS, Dove WF, Duyk G, Dymecki S, Eppig JT, Grieder FB, Heintz $\mathrm{N}$, Hicks $\mathrm{G}$, Insel TR, Joyner $\mathrm{A}$, Koller $\mathrm{BH}$, Lloyd $\mathrm{KC}$, Magnuson T, Moore MW, Nagy A, Pollock JD, Roses AD, Sands AT, Seed B, Skarnes WC, Snoddy J, Soriano P, Stewart DJ, Stewart F, Stillman B, Varmus H, Varticovski L, Verma IM, Vogt TF, von Melchner H, Witkowski J, Woychik RP, Wurst W, Yancopoulos GD, Young SG, Zambrowicz B. 2004. The knockout mouse project. Nat Genet 36:921-924.

Auwerx J, Avner P, Baldock R, Ballabio A, Balling R, Barbacid M, Berns A, Bradley A, Brown S, Carmeliet P, Chambon P, Cox R, Davidson D, Davies K, Duboule D, Forejt J, Granucci F, Hastie N, de Angelis MH, Jackson I, Kioussis D, Kollias G, Lathrop M, Lendahl U, Malumbres M, von Melchner H, Muller W, Partanen J, Ricciardi-Castagnoli P, Rigby P, Rosen B, Rosenthal N, Skarnes B, Stewart AF, Thornton J, Tocchini-Valentini G, Wagner E, Wahli W, Wurst W. 2004. The European dimension for the mouse genome mutagenesis program. Nat Genet 36:925-927.

Bentley A, MacLennan B, Calvo J, Dearolf CR. 2000. Targeted recovery of mutations in Drosophila. Genetics 156:1169-1173. 
Brown SD, Balling R. 2001. Systematic approaches to mouse mutagenesis. Curr Opin Genet Dev 11:268-273.

Cecchi C, Biasotto M, Tosi M, Avner P. 1997. The mottled mouse as a model for human Menkes disease: identification of mutations in the Atp7a gene. Hum Mol Genet 6:425-433.

Chen Y, Yee D, Dains K, Chatterjee A, Cavalcoli J, Schneider E, Om J, Woychik RP, Magnuson T. 2000. Genotype-based screen for ENU-induced mutations in mouse embryonic stem cells. Nat Genet 24:314-317.

Coghill EL, Hugill A, Parkinson N, Davison C, Glenister P, Clements S, Hunter J, Cox RD, Brown SD. 2002. A gene-driven approach to the identification of ENU mutants in the mouse. Nat Genet 30:255-256.

Colbert T, Till BJ, Tompa R, Reynolds S, Steine MN, Yeung AT, McCallum CM, Comai L, Henikoff S. 2001. High-throughput screening for induced point mutations. Plant Physiol 126:480-484.

Del Tito BJ, Jr, Poff HE, 3rd, Novotny MA, Cartledge DM, Walker RI, 2nd, Earl CD, Bailey AL. 1998. Automated fluorescent analysis procedure for enzymatic mutation detection. Clin Chem 44:731-739.

Edgley M, D'Souza A, Moulder G, McKay S, Shen B, Gilchrist E, Moerman D, Barstead R. 2002. Improved detection of small deletions in complex pools of DNA. Nucleic Acids Res 30:e52.

Engelke CE, Meinl W, Boeing H, Glatt H. 2000. Association between functional genetic polymorphisms of human sulfotransferases 1A1 and 1A2. Pharmacogenetics 10:163-169.

Glatt H. 2000. Sulfotransferases in the bioactivation of xenobiotics. Chem Biol Interact 129:141-170.

Glatt HR. 2002. Sulphotransferases. In: Ioannides C, editor. Handbook of enzyme systems that metabolise drugs and other xenobiotics. Sussex: John Wiley \& Sons. p 353-439.

Greber B, Lehrach H, Himmelbauer H. 2003. Characterization of trimethylpsoralen as a mutagen for mouse embryonic stem cells. Mutat Res 525:67-76.

Hogan B, Beddington R, Costantini F, Lacy E. 1994. Manipulating the mouse embryo: a laboratory manual. Cold Spring Harbor, NY: Cold Spring Harbor Laboratory Press. p 255-290.

Hooper M, Hardy K, Handyside A, Hunter S, Monk M. 1987. HPRT-deficient (Lesch-Nyhan) mouse embryos derived from germline colonization by cultured cells. Nature 326:292-295.

Jansen G, Hazendonk E, Thijssen KL, Plasterk RH. 1997. Reverse genetics by chemical mutagenesis in Caenorhabditis elegans. Nat Genet 17:119-121.

Justice MJ, Noveroske JK, Weber JS, Zheng B, Bradley A. 1999. Mouse ENU mutagenesis. Hum Mol Genet 8:1955-1963.

Krawczak M, Reiss J, Cooper DN. 1992. The mutational spectrum of single base-pair substitutions in mRNA splice junctions of human genes: causes and consequences. Hum Genet 90:41-54.

Li Q, Liu Z, Monroe H, Culiat CT. 2002. Integrated platform for detection of DNA sequence variants using capillary array electrophoresis. Electrophoresis 23:1499-1511.

Matise MP, Auerbach W, Joyner AL. 2000. Production of targeted embryonic stem cell clones. In: Joyner AL, editor. Gene targeting, a practical approach. Oxford: Oxford University Press. p 101-132.
McCallum CM, Comai L, Greene EA, Henikoff S. 2000. Targeted screening for induced mutations. Nat Biotechnol 18: 455-457.

Munroe RJ, Bergstrom RA, Zheng QY, Libby B, Smith R, John SW, Schimenti KJ, Browning VL, Schimenti JC. 2000. Mouse mutants from chemically mutagenized embryonic stem cells. Nat Genet 24:318-321.

Nadeau JH, Balling R, Barsh G, Beier D, Brown SD, Bucan M, Camper S, Carlson G, Copeland N, Eppig J, Fletcher C, Frankel WN, Ganten D, Goldowitz D, Goodnow C, Guenet JL, Hicks G, Hrabe de Angelis M, Jackson I, Jacob HJ, Jenkins N, Johnson D, Justice M, Kay S, Kinglsey D, Lehrach H, Magnuson T, Meisler M, Poustka A, Rinchik EM, Rossant J, Russell LB, Schimenti J, Shiroishi T, Skarnes WC, Soriano P, Stanford W, Takahashi JS, Wurst W, Zimmer A, International Mouse Mutagenesis Consortium. 2001. Sequence interpretation. Functional annotation of mouse genome sequences. Science 291:1251-1255.

O'Donovan MC, Oefner PJ, Roberts SC, Austin J, Hoogendoorn B, Guy C, Speight G, Upadhyaya M, Sommer SS, McGuffin P. 1998. Blind analysis of denaturing high-performance liquid chromatography as a tool for mutation detection. Genomics 52:44-49.

Oleykowski CA, Bronson Mullins CR, Godwin AK, Yeung AT. 1998. Mutation detection using a novel plant endonuclease. Nucleic Acids Res 26:4597-4602.

Smits BM, Mudde J, Plasterk RH, Cuppen E. 2004. Target-selected mutagenesis of the rat. Genomics 83:332-334.

Stanford WL, Cohn JB, Cordes SP. 2001. Gene-trap mutagenesis: past, present and beyond. Nat Rev Genet 2: 756-768.

Stanssens P, Zabeau M, Meersseman G, Remes G, Gansemans Y, Storm N, Hartmer R, Honisch C, Rodi CP, Bocker S, van den Boom D. 2004. High-throughput MALDI-TOF discovery of genomic sequence polymorphisms. Genome Res $14: 126-133$.

Vivian JL, Chen Y, Yee D, Schneider E, Magnuson T. 2002. An allelic series of mutations in $\mathrm{Smad} 2$ and $\mathrm{Smad} 4$ identified in a genotype-based screen of $\mathrm{N}$-ethyl-N- nitrosourea-mutagenized mouse embryonic stem cells. Proc Natl Acad Sci USA 99:15542-15547.

Wienholds E, Van Eeden F, Kosters M, Mudde J, Plasterk RH, Cuppen E. 2003. Efficient target-selected mutagenesis in zebrafish. Genome Res 13:2700-2707.

Yang B, Wen X, Kodali NS, Oleykowski CA, Miller CG, Kulinski J, Besack D, Yeung JA, Kowalski D, Yeung AT. 2000. Purification, cloning, and characterization of the CEL I nuclease. Biochemistry 39:3533-3541.

Zan Y, Haag JD, Chen KS, Shepel LA, Wigington D, Wang YR, $\mathrm{Hu}$ R, Lopez-Guajardo CC, Brose HL, Poerter KI, Leonard RA, Hitt AA, Schommer SL, Elegbede AF, Gould MN. 2003. Production of knockout rats using ENU mutagenesis and a yeast-based screening assay. Nat Biotechnol 21:645-651. 